so dass eher die perzipierte Berichterstattung als die persönliche Einstellung gemessen wird.

Als letztes präsentieren die Autoren Befunde aus offenen Interviews mit vier leitenden Nachrichtenredakteuren, zwei der Tagesschau und zwei von RTL-aktuell. Da zwei der Gesprächspartner im Text namentlich genannt werden, ist davon auszugehen, dass sie einer nicht anonymisierten Ergebnispräsentation zustimmten, was wiederum Auswirkungen auf ihre Antworten gehabt haben könnte. Die Auswertung des Interviewmaterials orientiert sich an den $\mathrm{Di}$ mensionen von Journalismuskultur nach $\mathrm{Ha}$ nitzsch und ergibt, dass es hier keine allzu markanten Unterschiede zwischen den Repräsentanten des öffentlich-rechtlichen und des privaten Senders gibt. Bei insgesamt nur vier Fällen ist dieser Befund freilich nicht verallgemeinerbar.

Die Untersuchung wartet insgesamt gesehen mit vielen interessanten Befunden auf und aktualisiert vor allem den Forschungsstand zu inhaltlichen Strukturen und Publikumsinteresse an Auslandsberichterstattung. Besonders hervorzuheben ist, dass die deutschen Befunde an passenden Stellen in den Kontext der internationalen Studie gestellt werden, was eine bessere Einordnung der Ergebnisse erlaubt. Die leise Kritik, die man an der Studie äußern kann, bezieht sich auf ihre theoretische Fundierung: Sie verfolgt keine übergreifenden, theoretisch-erklärenden Ansprüche. Beispielsweise wäre es denkbar gewesen, bestimmte Befunde, zum Beispiel zur Nachrichtengeografie, systematisch aus der Perspektive der Globalisierung oder Westernisierung zu deuten. Auch wird bestimmten, unerwarteten Befunden wie z. B. dem geringen Vorkommen Frankreichs in der Berichterstattung - immerhin engster politischer und ökonomischer Partner Deutschlands mit traditionell starker Präsenz in der Berichterstattung - zu wenig Beachtung geschenkt. Bezeichnenderweise zeigten sich die Redakteure, die u. a. mit diesem Ergebnis in der Befragung konfrontiert wurden, davon auch besonders überrascht. $\mathrm{Ob}$ es sich jedoch dabei sowie bei der im Vergleich dazu deutlich stärkeren Ausrichtung der Nachrichten auf Regionen wie Großbritannien oder den Nahen Osten um eine dauerhafte Verschiebung medialer Prioritäten handelt oder um eine situationsbedingte Momentaufnahme, wird nicht besprochen. Daher bleibt am Ende festzuhalten, dass der theoretische Wert der Untersuchung begrenzt ist, obgleich sie eine Fülle an Informationen enthält, die ein breites Spektrum an Lesern interessieren dürfte.

Peter Maurer

\section{Christian Rauda}

\section{Recht der Computerspiele}

München: C.H. Beck, 2013. - 318 S.

ISBN 978-3-406-64938-7

Seit einigen Jahren wächst die Bedeutung rechtlicher Fragen rund um Computerspiele. Führte die Branche bislang ein eher bescheidenes $\mathrm{Da}$ sein, abseits des großen IT-Marktes, wird der Markt derzeit erstaunlich groß - verbunden mit einem proportionalen Wachstum an schwierigen rechtsdogmatischen Fragen. Es ist daher ein besonderes Verdienst von Herrn Rauda, sich dieses schwierigen Rechtsgebietes angenommen zu haben. Christian Rauda ist Rechtsanwalt, Zauberkünstler und Justiziar des Deutschen Internetverbandes. Er beginnt seine Studie mit einem Überblick über elektronische Spiele, differenziert nach Plattformen und Genres. Diese ökonomische Vorüberlegung hält sich seitenmäßig in Grenzen, so dass der Verfasser schon sehr schnell auf ein erstes großes Rechtsgebiet, nämlich das Urheberrecht, zu sprechen kommen kann (S. 12ff.). Man merkt ihm hier an, dass der Verfasser sehr pro Computerspielindustrie argumentiert. So will er die bisherige Rechtsprechung zum Fabelschutz (entwickelt für literarische Texte) auf den Schutz von Spielideen beziehen (Rn. 40). Diesen Fabelschutz will er auch auf die Spielregeln beziehen (Ziffer 65). Auf diese Weise will er Programmierer und Game-Designer gleichstellen (Ziffer 69). Er erläutert dann den Schutz von Software, ohne allerdings klarer zwischen Software und Computerspielen abzugrenzen (Rn. 70ff). Für mich neu waren die Überlegungen zum sog. Balancing, dem inneren Gleichgewicht des Spiels (S. 18ff.), das der Verfasser urheberrechtlich schützen möchte.

Abgelehnt wird ein Schutz des BildschirmDisplays über den Fotografienschutz (S. 33f.). Für das Computerspiel insgesamt wird der Laufbildschutz nach $\$ \$ 95,94$ UrhG geprüft (S.39ff.). Hinzu kommen Überlegungen zur Musik, zu Sprachaufnahmen und zum Schutz von Computerspielen als Laufbild (letzteres ab Rn. 103). Knapp geraten sind die Hinweise zum Urhebervertragsrecht, insbesondere was den Schutz von Urhebern nach $\$ \$ \$ 32,32$ a UrhG oder AGB-rechtliche Beschränkungen bei Standardverträgen angeht (Rn.108ff.). Die Gliederung ist oft nicht ganz stimmig, wie man an Rn. 117 sehen kann, wo die Überschrift „4. Auftragsproduktion" als ersten Unterpunkt den Gliederungspunkt „c) echte Auftragsproduktion " trägt. Erstaunlich breit angelegt ist der Abschnitt zum Rückruf von Nutzungsrechten 
wegen Nichtausübung (Rn. 133ff.). Allerdings merkt man hier noch eine weitere Charakteristik des Werkes, nämlich die klare Akzentuierung auf Rechtsprechung. So wird in Ziffer 137 die BGH-Rechtsprechung zu den Auswirkungen eines Rückrufs auf Unterlizenzen geschildert, ohne entsprechende Kritikpunkte anzubringen.

Kurz abgehandelt werden auch die Urheberpersönlichkeitsrechte (Rn.138ff.), gefolgt von Hinweisen zur freien Benutzung und zum $\mathrm{Zi}$ tatrecht (letzteres ab Rn. 159). Als „Hardliner" tritt der Verfasser bei der Frage der Urheberrechtsverletzungen im Internet auf. Hinweise zum Streaming findet man hier nicht. Auch ist P2P offensichtlich kein Thema mehr, denn ansonsten würde der Verfasser erkennen, dass es bei solchen Systemen gar nicht um die Vervielfältigung, sondern um das Bereitstellen für die Öffentlichkeit geht (anders Ziffer 175). Knapp geraten sind auch die Hinweise zum Host-Provider (ab Rn.189). Die Rechtsprechung hierzu mag kompliziert sein, reduziert sich aber nicht nur auf die Frage der Haftung von RapidShare (so ab Rn. 197). Das Problem, ob Computerspiele unter $\$ 95 \mathrm{a}$ oder $\$ 69 \mathrm{f}$ UrhG fallen, hat der $\mathrm{BGH}$ nicht gesehen (Rn. 202), obwohl der BGH diese spannende Frage nun gerade dem EuGH zur Entscheidung vorgelegt hat.

Im nächsten Kapitel geht es dem Verfasser um das Wettbewerbsrecht, konkret um den wettbewerbsrechtlichen Nachahmungsschutz (ab Rn. 208). Hier schildert der Verfasser insbesondere die Rechtsprechung des Landgerichts Köln zur wettbewerblichen Eigenart von Web-Sites (Rn. 212) sowie die Rechtsprechung des OLG Frankfurt zum Computerspiel Donkey Kong Junior (Rn. 215). Eine weitere Fallgruppe des UWG sieht der Verfasser im Schutz gegen Eingriffe in das Spielgeschehen (Rn. 225). Hier geht der Verfasser auf einige Entscheidungen aus der Entscheidungspraxis des Landgerichts Hamburg ein, etwa im Fall von Cheatbots und des Farming (letzteres ab Rn. 233). In weiteren Abschnitten finden sich Ausführungen zum Patentschutz (Rn. 236ff.), zum Titel und Markenschutz (letzteres ab Rn. 275) sowie zum Geschmacksmusterrecht (Rn. 130ff.). Viel Neues findet sich darin nicht, ebenso nicht wie in dem Kapitel zum Domainrecht (Rn. 371) oder bei der Frage der Gerichtszuständigkeit (Rn. 410ff.). Hier übersieht der Verfasser auch noch das Gesetz über unseriöse Geschäftspraktiken, das gerade im Bereich Urheberrecht den fliegenden Gerichtsstand zugunsten von Verbrauchern aufgehoben hat (Rn. 412). Hier wirkt die Arbeit recht grob, etwa wenn der Verfasser empfiehlt, bei Verletzungen durch außereuropäische Verletzer das Übereinkommen von Lugano anzuwenden (Rn. 411), das sicherlich nicht gegen alle außereuropäischen Verletzer hilft.

Die Erstellung dieses Buches dürfte eine Mordsarbeit gewesen sein. Denn der Verfasser schlängelt sich durch alle Rechtsgebiete hindurch und schafft so dem Leser auf knapp 300 Seiten eine sehr gute Übersicht über die verschiedenen Facetten des Computerspielerechts. In weiten Teilen trägt die Arbeit allerdings den Charakter eines Leitfadens für die einschlägige Spieleszene. Für den wissenschaftlich interessierten Leser sind die Passagen am interessantesten, in denen der Verfasser seine schon herausragende Tatsachenkenntnis vom Spielemarkt in die Arbeit einbringt. Von daher ist die Lektüre des Buchs auf jeden Fall gewinnbringend. Dem Verfasser sei eine breite Akzeptanz und viele neue Auflagen gewünscht.

Thomas Hoeren

\section{Anne Schulze}

\section{Internetwerbung und Kinder}

Eine Rezeptionsanalyse

Heidelberg: Springer VS, 2013. - 248 S.

ISBN 978-3-658-02829-9

(Zugl.: Münster, Univ., Diss., 2012)

Dass Kinder längst eine explizit und mit vielfältigen Konzepten angesprochene Zielgruppe von Werbung sind, führt immer wieder zu teils hitzigen, ideologiegesättigten Debatten. Dass in einem durchkommerzialisierten, dem kapitalistischen Verwertungsprinzip unterworfenen Mediensystem „kostenlose“ Angebote nur möglich sind, wenn die erhofften Gewinne anderweitig erwirtschaftet werden, wird dabei gerne ausgeblendet.

Anne Schulze wendet sich einer aktuellen Ausprägung des Spannungsfeldes „Kinder und Werbung “ zu. In ihrer Dissertation untersucht sie mit einem Fokus auf die Erhebung von „Internetwerbekompetenz" den Umgang von Kindern mit im Internet geschalteter Werbung. Der Schwerpunkt der Untersuchung liegt dabei auf den eher „traditionellen“ Formen der Internetwerbung (Werbebanner, Pop-Ups und Internetseiten wie de.barbie.com). Die neueren Formen von Werbung, wie sie gerade auch im Internet zu finden sind (z.B. „virale“ Werbung), werden dagegen nicht thematisiert.

Die Verfasserin geht das Thema breit an. Die ersten drei Kapitel (und damit nahezu die Hälfte des Bandes) dienen der Aufarbeitung der bis- 